\title{
Role of Rectal Diclofenac Suppository for Prevention and Its Impact on Severity of Post-Endoscopic Retrograde Cholangiopancreatography Pancreatitis in High-Risk Patients
}

\author{
Sandeep Patil ${ }^{\mathrm{a}}$, Vikas Pandey ${ }^{\mathrm{a}, \mathrm{b}}$, Nilesh Pandav ${ }^{\mathrm{a}}$, Meghraj Ingle ${ }^{\mathrm{a}}$, Aniruddha Phadke ${ }^{\mathrm{a}}$, \\ Prabha Sawant ${ }^{\mathrm{a}}$
}

\begin{abstract}
Background: The aim was to study the role of rectal diclofenac in prevention of post-endoscopic retrograde cholangiopancreatography (ERCP) pancreatitis and its impact on severity of post-ERCP pancreatitis.

Methods: We conducted a single-center, prospective, open-labeled, randomized trial for evaluating the use of rectal diclofenac in prevention of post-ERCP pancreatitis in high-risk patients. We assessed 526 patients coming for ERCP for different indications. Four hundred patients were eligible for the study. Those not fitting the high-risk criteria and with acute pancreatitis were excluded. These patients were randomized in two groups: 200 patients received rectal diclofenac prior to or during the procedure, while 200 patients received placebos. Serum amylase was measured at 2 and 36 hours. Post-ERCP pancreatitis was defined as serum amylase $>3$ times upper limit of normal with associated severe abdominal pain. Severity was graded according to days of hospitalization and complications.
\end{abstract}

Results: Twenty-nine out of $400(7.2 \%)$ patients developed postERCP pancreatitis. Six out of $200(3 \%)$ patients in rectal diclofenac group developed post-ERCP pancreatitis compared to 23 out of 200 $(11.5 \%)$ patients in placebo group. The difference was statistically significant $(\mathrm{P}=0.001)$. All patients $(\mathrm{six})$ in rectal diclofenac group developed mild pancreatitis as compared to severe pancreatitis in four and moderate pancreatitis in five patients in the placebo group.

Conclusion: Rectal diclofenac prior to or during ERCP in high-risk patients reduces the incidence as well as severity of post-ERCP pancreatitis compared to placebo.

Keywords: Rectal; Diclofenac; Prevention; Pancreatitis

Manuscript accepted for publication September 18, 2015

${ }^{a}$ Department of Gastroenterology, Lokmanya Tilak Municipal Medical College \& General Hospital, Mumbai 400022, India

${ }^{\mathrm{b}}$ Corresponding Author: Vikas Pandey, Department of Gastroenterology, Lokmanya Tilak Municipal Medical College \& General Hospital, Mumbai 400022, India. Email: reenaupadhyay66@gmail.com

doi: http://dx.doi.org/10.14740/gr672w

\section{Introduction}

Pancreatitis is a major well-known complication of endoscopic retrograde cholangiopancreatography (ERCP) with reported incidence ranging from $1 \%$ to $10 \%$ in various series [1-3]. It can cause significant morbidity and occasional deaths are also reported. The risk factors for post-ERCP pancreatitis are well known [4]. Various theories about pathogenesis of post-ERCP pancreatitis have been proposed. But the most accepted theory is mechanical trauma to papilla or pancreatic sphincter causing transient obstruction to outflow of pancreatic juice. Another theory suggests the increased hydrostatic pressure in pancreatic duct caused by injection of contrast or saline cause injury to parenchyma. Regardless of mechanism, the cascade of events is initiated resulting in activation of proteolytic enzymes causing autodigestion of pancreas and impaired acinar secretion. This results in activation of inflammatory cascade causing both local inflammation and systemic effects $[5,6]$. The interventions for prevention of post-ERCP pancreatitis aim at breaking this cascade. Non-steroidal anti-inflammatory drugs (NSAIDs) are potent inhibitor of phospholipase A2 which is thought to play a critical role in early inflammatory cascade [7]. Rectal diclofenac is a cheap, widely available agent with easy method of administration and favorable side effect profile makes it an attractive option. There are limited data on efficacy of NSAIDs in prevention of post-ERCP pancreatitis. Rectal diclofenac has been evaluated in few trials earlier but most trials included low-risk patients and sample size of these trials was very small. Till date, sparse Indian data are available which evaluated rectal diclofenac in prevention of post-ERCP pancreatitis. We conducted a prospective, single-center, open-labeled, randomized placebo-controlled trial evaluating role of rectal diclofenac in prevention of post-ERCP pancreatitis in high-risk patients and whether it has any implications on severity of post-ERCP pancreatitis.

\section{Patients and Methods}

This study was performed at a tertiary care center between $\mathrm{Au}$ gust 2011 and June 2014. We enrolled 526 patients that were referred for ERCP for different indications (Fig. 1). Inclusion criteria involved only those patients with high risk of develop- 


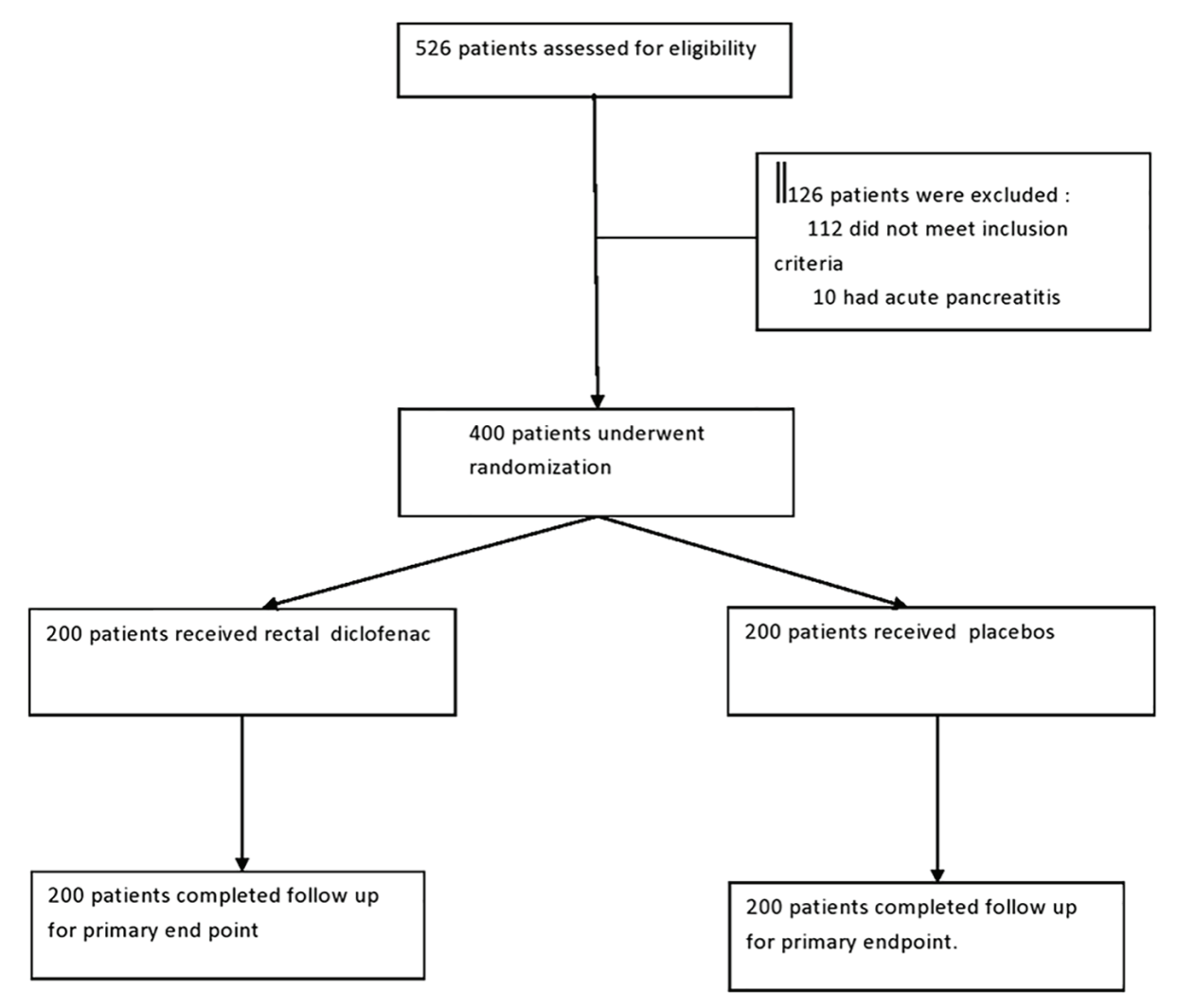

Figure 1. Patients flow chart.

ing post-ERCP pancreatitis. Patients were considered having high risk of development of post-ERCP pancreatitis if they had one or more major risk factors: suspected sphincter of Oddi dysfunction (SOD), prior history of post-ERCP pancreatitis, difficult or failed cannulation (more than five attempts), repeated pancreatic cannulation, pancreatic duct injection with acinarization, pancreatic sphincterotomy, precut sphincterotomy, biliary sphincterotomy for suspected SOD, and ampullectomy; or if they had two or more minor risk factors for development of post-ERCP pancreatitis: female gender, young age, history of recurrent acute pancreatitis, normal serum bilirubin, lack of choledocholithiasis, pancreatic brush cytology, and balloon dilatation of intact biliary sphincter. Those patients not fitting the high-risk criteria, acute pancreatitis at the time of ERCP, contraindications to the use of NSAIDs (active peptic ulcer disease or S. creatinine $>1.4 \mathrm{mg} / \mathrm{dL}$ ), and who had ingested NSAIDs in last 1 week were excluded from the study. Four hundred patients were eligible for the study. One hundred twenty-six patients were excluded from the study depending on the exclusion criteria. These patients were randomized in two groups each containing 200 patients. Randomization was done in 1:1 ratio by computergenerated method. It was balanced in random blocks of five patients. One group $(n=200)$ received rectal diclofenac suppository (containing $100 \mathrm{mg}$ of diclofenac) immediately prior to or during the procedure. Other group $(n=200)$ received glycerine suppositories as placebos. ERCP was performed under sedation with intravenous midazolam by two experienced operators. Injection hyoscine was given for control of bowel motility. During the procedure, an assistant recorded the details of the procedure like timing of procedure, number of pancreatic duct cannulation and injection, difficulty in cannulation, and whether precut, pancreatic sphincterotomy, and balloon sphincteroplasty were done. Patients in rectal diclofenac or placebo group received rectal suppository immediately prior to or during the procedure. Pancreatic duct stents were placed in those patients in which pancreatic cannulation occurred more than two times or pancreatic injection with contrast or saline during the procedure. Maximum procedure time for the ERCP was $70 \mathrm{~min}$. Post-procedure patients were admitted for observation. Patients were assessed for any immediate complications, abdominal pain, and distention. Patients were subjected to testing of serum amylase at 2 and $32 \mathrm{~h}$ post-procedure. Those patients who had no abdominal pain, vomiting, back pain and $2 \mathrm{~h}$ serum amylase levels less than two times upper limit of normal were started on oral liquids 3 - $4 \mathrm{~h}$ after ERCP. Post-ERCP pancreatitis was defined as rise in serum amylase more than three times upper limit of normal $24 \mathrm{~h}$ after ERCP with associated clinical feature of severe abdominal pain requiring persistent hospitalization. Primary endpoint of the study was to detect number of patients developing post-ERCP pancreatitis in both groups. Those patients diagnosed as post-ERCP pancreatitis were kept hospitalized. These patients received intravenous antibiotics, supportive treatment for pancreatitis. Patients were subjected to routine biochemical investigations, imaging modalities like ultrasound abdomen and contrast-enhanced computed tomography to detect complications of pancreatitis. The severity of pancreatitis was graded as 
Table 1. Patient Details About Indication of ERCP

\begin{tabular}{|c|c|c|c|}
\hline Indication of ERCP & $\begin{array}{l}\text { Rectal diclofenac group } \\
(n=200)\end{array}$ & $\begin{array}{l}\text { Placebo group } \\
(\mathrm{n}=200)\end{array}$ & P value \\
\hline Gallbladder stones (with dilated CBD) & 25 & 27 & $>0.05$ \\
\hline Common bile duct stones & 52 & 57 & $>0.05$ \\
\hline Post cholecystectomy & 20 & 18 & $>0.05$ \\
\hline Malignancy (gallbladder, periampullary, cholangiocarcinoma) & 21 & 16 & $>0.05$ \\
\hline ERCP + suspected sphincter of Oddi dysfunction & 66 & 69 & $>0.05$ \\
\hline Common bile duct stricture (including those with chronic pancreatitis) & 16 & 13 & $>0.05$ \\
\hline
\end{tabular}

mild, moderate and severe according to days of hospitalization required and complications of pancreatitis. Mild post-ERCP pancreatitis was defined as requiring an unplanned admission or prolongation of hospitalization by 2 - 3 days. Moderate postERCP pancreatitis was defined as requiring hospitalization of 4 - 10 days and severe post-ERCP pancreatitis was defined as requiring hospitalization of greater than 10 days or requiring intensive care or intervention for local complications of pancreatitis. The secondary endpoint of the study was to assess the severity of post-ERCP pancreatitis in both groups.

\section{Statistical analysis}

For the analysis of primary endpoint, we used Fisher exact test to analyze the difference in proportion of patients with postERCP pancreatitis in rectal diclofenac and placebo groups with $\mathrm{P}$ value $<0.05$ indicating significant difference. Patients' demographic and clinical factors were compared using Fisher exact test or $\mathrm{X}^{2}$ test as appropriate.

\section{Results}

A total of 400 patients entered the study; 200 patients received rectal diclofenac, while 200 patients received glycerine suppository (control group). There were 128 (64\%) female patients in rectal diclofenac group while $123(61.5 \%)$ in placebo group. The mean age in rectal diclofenac group was 45.44 years while in placebo group was 47.86 years. The patients in both groups were well matched for the indication of ERCP as depicted in Table 1. We compared various risk factors prior to or during the procedure which might increase the risk of post-ERCP pancreatitis like pancreatic cannulation, precut sphincterotomy, suspected SOD, pancreatic sphincterotomy, pancreatic duct injection, balloon sphincteroplasty in patients with suspected SOD, and difficulty of failed cannulation in both groups. The incidences of these various risk factors were similar in both the groups. The baseline characteristics of the patients in both groups were identical as depicted in Table 2. A total of 23 patients, 12 in placebo group and 11 patients in rectal diclofenac group, received pancreatic stents due to recurrent pancreatic duct cannulation or pancreatic duct injection with acinarization. Incidence of post-ERCP pancreatitis was compared in both groups. Twenty-nine out of $400(7.2 \%)$ patients developed post-ERCP pancreatitis. Six out of $200(3 \%)$ patients in rectal diclofenac group developed post-ERCP pancreatitis compared to 23 out of $200(11.5 \%)$ patients in placebo group (Fig. 2). Two-tailed Fisher exact test was applied. The difference was statistically significant with $\mathrm{P}$ value $<0.05$. In

Table 2. Characteristic of Patients at Baseline

\begin{tabular}{llll}
\hline Characteristic & Rectal diclofenac group $(\mathbf{n}=\mathbf{2 0 0})$ & Placebo group $(\mathbf{n}=\mathbf{2 0 0})$ & P value \\
\hline Mean age, years & 45.44 & 47.86 & $>0.05$ \\
Female sex, no. (\%) & $128(64 \%)$ & $123(61.5 \%)$ & $>0.05$ \\
Suspected sphincter of Oddi dysfunction (\%) & $66(33 \%)$ & $69(34.5 \%)$ & $>0.05$ \\
Precut sphincterotomy (\%) & $21(10.5 \%)$ & $24(12 \%)$ & $>0.05$ \\
Pancreatic duct cannulation (\%) & $26(13 \%)$ & $24(12 \%)$ & $>0.05$ \\
Pancreatic acinarization (\%) & $9(4.5 \%)$ & $8(4 \%)$ & $>0.05$ \\
History of recurrent pancreatitis (\%) & $40(20 \%)$ & $35(17.5 \%)$ & $>0.05$ \\
History of post ERCP pancreatitis (\%) & $6(3 \%)$ & $5(2.5 \%)$ & $>0.05$ \\
Pancreatic sphincterotomy (\%) & $11(5.5 \%)$ & $12(6 \%)$ & $>0.05$ \\
Ampullectomy & None & None & $>0.05$ \\
Difficult cannulation (\%) & $61(30.5 \%)$ & $66(33 \%)$ & $>0.05$ \\
Pancreatic duct stenting (\%) & $11(5.5 \%)$ & $12(6 \%)$ & $>05$ \\
\hline
\end{tabular}




\section{Post ERCP pancreatitis patients}

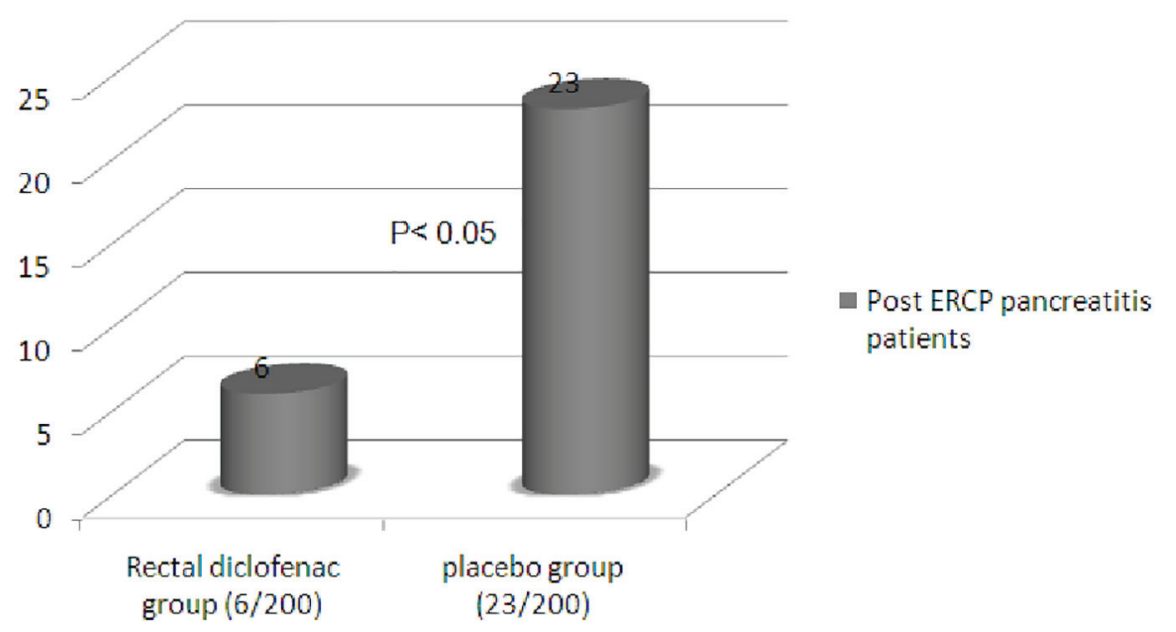

Figure 2. Post-ERCP pancreatitis cases.

a subgroup analysis, incidence of post-ERCP pancreatitis in patients with suspected SOD in rectal diclofenac group was $6 \%(4 / 66)$ while in placebo group was $18.8 \%$ (13/69). Among patients who received pancreatic stent, only one patient in rectal diclofenac group (1/11) developed post-ERCP pancreatitis compared to two patients in placebo group (2/12); however, the number of patients in which pancreatic stenting was performed was low in our study. The secondary endpoint of the study was to assess the severity of post-ERCP pancreatitis in both groups. The severity of post-ERCP pancreatitis in rectal diclofenac group was mild in all six patients $(100 \%)$, while in placebo group, it was severe in four patients $(4 / 23,17.3 \%)$, moderate in five patients $(5 / 23,21.7 \%)$ and mild in 14 pa- tients $(14 / 23,60.8 \%)$ (Fig. 3$)$. The difference in severity of pancreatitis in rectal diclofenac and placebo groups was statistically significant. Two out of four patients in placebo group who had severe post-ERCP pancreatitis developed pancreatic pseudocyst, but were managed conservatively. One out of four patients in placebo group with severe post-ERCP pancreatitis developed right-sided pleural effusion requiring therapeutic pleural tapping. One out of four patients in placebo group with severe post-ERCP pancreatitis developed subacute intestinal obstruction. Two deaths occurred in the placebo group, both with severe post-ERCP pancreatitis, one with right-sided pleural effusion and the other with subacute intestinal obstruction. Adverse events like bleeding while ERCP in sphincteromized

\section{SEVERITY OF POST ERCP PANCREATITIS}

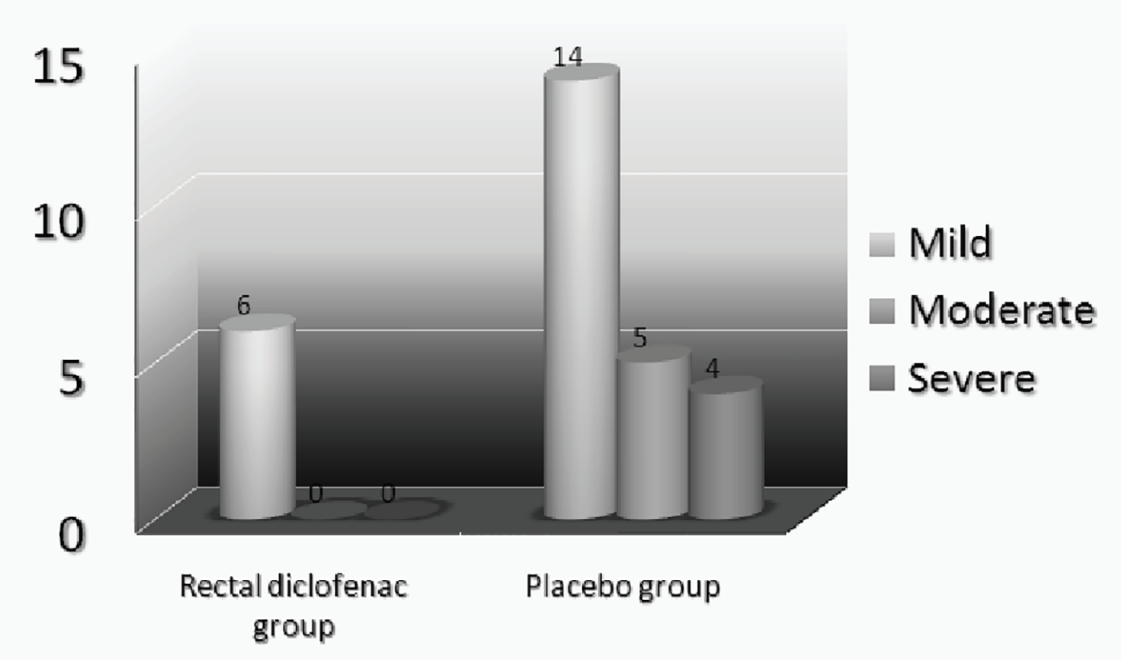

Figure 3. Severity of post-ERCP pancreatitis. 
patients were noted in 16 patients, six in placebo group and eight patients in rectal diclofenac group but responded to adrenaline injection and coagulation. All of these patients had minor bleeding and did not require blood transfusion. Rectal diclofenac suppository was well tolerated in all the patients with no adverse event noted with use of rectal diclofenac.

\section{Discussion}

Our study has shown that single dose administration of rectal diclofenac prior to or during the procedure has reduced the incidence of post-ERCP pancreatitis. It also has profound impact on reducing the severity of post-ERCP pancreatitis. Majority of our patients had suspected SOD. In subgroup analysis, our study has shown that rectal diclofenac was also effective in this group of patients in reducing post-ERCP pancreatitis. Rectal diclofenac is a cheap drug, easily available and with a favorable side effect profile. It is underutilized in routine clinical practice. Pancreatic stents are of proven benefit in preventing post-ERCP pancreatitis $[8,9]$ but difficult to use in routine practice because of difficulty in pancreatic duct cannulation and requirement of operator expertise. Comparatively rectal diclofenac is very easy to administer and is easily available. The mechanism of post-ERCP pancreatitis is not clearly understood. But most accepted theory is trauma of thermal injury to papilla causing transient obstruction to pancreatic secretion. Another accepted theory is hydrostatic pressure due to contrast or saline injection into pancreatic duct. This injury initiates an inflammatory cascade leading to activation of proteolytic enzymes intraductally causing pancreatic autodigestion and impaired secretion. Diclofenac inhibits phospholipase A2 which is an important mediator of various reactions in this inflammatory cascade breaking this cycle $[5,6]$. Peak concentration of rectal diclofenac occurs between 30 and 90 min after insertion with complete bioavailability. The elimination half life is $2 \mathrm{~h}$. Rectal diclofenac has been evaluated in different randomized clinical trials. In a study by Murray et al [10], 220 patients with high risk of post-ERCP pancreatitis were randomized in two groups, rectal diclofenac vs. placebo. In these patients rectal diclofenac was given immediately after the procedure. There was significant reduction in incidence of post-ERCP pancreatitis in rectal diclofenac group as compared to placebo which was similar to our study. However, in this study, there was no significant difference in patients with SOD in rectal diclofenac vs. placebo group. Our study demonstrated that there is significant reduction in incidence of post-ERCP pancreatitis even in patients with suspected SOD. Khoshbaten et al [11] evaluated the use of rectal diclofenac in patients with extrahepatic cholestasis undergoing ERCP. This study included 100 patients which were randomized into rectal diclofenac and placebo groups. This study also reported significant reduction in incidence of post-ERCP pancreatitis in rectal diclofenac group similar to our study. However, this study has reported very high incidence of post-ERCP pancreatitis $(26 \%)$ in the control group. Oral diclofenac has been evaluated for prevention of post-ERCP pancreatitis by Cheon et al, but was found to be of no benefit [12]. Though rectal diclofenac is such an attractive option in prevention of post-ERCP pancreatitis, it is still underutilized. There are very few trials evaluating its use in prevention of post-ERCP pancreatitis. There are sparse Indian data available till date. This prospective single-center, open-labeled, randomized placebo-controlled trial has shown that use of single dose of rectal diclofenac immediately prior to or during the ERCP in high-risk patients is effective in not only reducing the incidence but also severity of post-ERCP pancreatitis. It is also effective in patients with suspected SOD. The major limitation of this study was severity of pancreatitis was graded according to the consensus guideline depending on days of hospitalization and complications. Various scoring systems like Ranson's score and APACHE II score were not utilized. However, similar guidelines were followed in previous studies.

\section{Conclusion}

Rectal diclofenac prior to or during ERCP in high-risk patients reduces the incidence as well as severity of post-ERCP pancreatitis compared to placebo.

\section{References}

1. Nebel OT, Silvis SE, Rogers G, Sugawa C, Mandelstam P. Complications associated with endoscopic retrograde cholangiopancreatography. Results of the 1974 A/S/G/E survey. Gastrointest Endosc. 1975;22(1):34-36.

2. LaFerla G, Gordon S, Archibald M, Murray WR. Hyperamylasaemia and acute pancreatitis following endoscopic retrograde cholangiopancreatography. Pancreas. 1986;1(2):160-163.

3. Rabenstein T, Hahn EG. Post-ERCP pancreatitis: new momentum. Endoscopy. 2002;34(4):325-329.

4. Freeman ML, DiSario JA, Nelson DB, Fennerty MB, Lee JG, Bjorkman DJ, Overby CS, et al. Risk factors for postERCP pancreatitis: a prospective, multicenter study. Gastrointest Endosc. 2001;54(4):425-434.

5. Messmann H, Vogt W, Holstege A, Lock G, Heinisch A, von Furstenberg A, Leser HG, et al. Post-ERP pancreatitis as a model for cytokine induced acute phase response in acute pancreatitis. Gut. 1997;40(1):80-85.

6. Karne S, Gorelick FS. Etiopathogenesis of acute pancreatitis. Surg Clin North Am. 1999;79(4):699-710.

7. Gross V, Leser HG, Heinisch A, Scholmerich J. Inflammatory mediators and cytokines - new aspects of the pathophysiology and assessment of severity of acute pancreatitis? Hepatogastroenterology. 1993;40(6):522-530.

8. Fazel A, Quadri A, Catalano MF, Meyerson SM, Geenen JE. Does a pancreatic duct stent prevent post-ERCP pancreatitis? A prospective randomized study. Gastrointest Endosc. 2003;57(3):291-294.

9. Freeman ML. Pancreatic stents for prevention of post-endoscopic retrograde cholangiopancreatography pancreatitis. Clin Gastroenterol Hepatol. 2007;5(11):1354-1365.

10. Murray B, Carter R, Imrie C, Evans S, O'Suilleabhain C. Diclofenac reduces the incidence of acute pancreatitis 
after endoscopic retrograde cholangiopancreatography. Gastroenterology. 2003;124(7):1786-1791.

11. Khoshbaten M, Khorram H, Madad L, Ehsani Ardakani MJ, Farzin H, Zali MR. Role of diclofenac in reducing post-endoscopic retrograde cholangiopancreatography pancreatitis. J Gastroenterol Hepatol. 2008;23(7 Pt 2):e11-16.

12. Cheon YK, Cho KB, Watkins JL, McHenry L, Fogel EL, Sherman S, Schmidt S, et al. Efficacy of diclofenac in the prevention of post-ERCP pancreatitis in predominantly high-risk patients: a randomized double-blind prospective trial. Gastrointest Endosc. 2007;66(6):1126-1132. 